\title{
Stage IVB Hilar Cholangiocarcinoma AJCC v7
}

National Cancer Institute

\section{Source}

National Cancer Institute. Stage IVB Hilar Cholangiocarcinoma A/CC v7. NCI Thesaurus. Code C88064.

Stage IVB includes: (Any T, N2, M0); (Any T, Any N, M1). N2: Metastasls to periaortic, pericaval, superior mesenteric artery, and/or celiac artery lymph nodes. M0: No distant metastasis. M1: Distant metastasis. (from AJCC 7th Ed.) 